\title{
Effect of Acupressure Sessions on Dyspnea and Fatigue among Patients with Bronchial Asthma
}

\author{
Rasha Aly Yakout ${ }^{1}$ \& Eshrak Salama Hashem ${ }^{2}$. \\ 1. Assist professor of Medical- Surgical Nursing Department, Faculty of Nursing, Alexandria University, Egypt. \\ 2. Lecturer of Medical- Surgical Nursing Department, Faculty of Nursing, Alexandria University, Egypt.
}

\begin{abstract}
Bronchial asthma is a syndrome characterized by airflow obstruction; Dyspnea and fatigue are the most disabling symptoms in asthma and the trigger point of physical inactivity with a consequent lower standard of quality of life. Acupressure is one of important non-pharmacological modalities which leads to reduction of asthma symptoms and ultimately enhances asthma control. This study aimed to evaluate the effect of acupressure sessions on dyspnea and fatigue among patients with bronchial asthma. Research design: A quasi experimental research design was conducted on sixty patients with bronchial asthma who were admitted to the Chest inpatient Unit of Alexandria Main University Hospital. Three tools were used for data collection: Tool I: Patients with bronchial asthma assessment. Tool II: Dyspnea Assessment questionnaire. Tool III: Fatigue Assessment questionnaire. Results of the present study revealed that there were significant differences between control and study group patients related to their grade of dyspnea and fatigue severity after application of acupressure sessions. Conclusion: acupressure may be effective as an adjuvant therapy to the traditional management for patients with bronchial asthma to improve dyspnea and fatigue syndromes. Recommendations of this study include, illustrated self-administered acupressure intervention booklets distributed to patients with bronchial asthma, could be of help, additionally, development and implementation of acupressure training program for nurses, about acupressure are advocated.
\end{abstract}

\section{Keywords: Acupressure, Bronchial Asthma, Dyspnea \& Fatigue.}

\section{Introduction}

Bronchial asthma is a syndrome characterized by airflow obstruction that varies markedly, both spontaneously and with treatment. Patients with bronchial asthma harbor a special type of airway inflammation that makes them more responsive to a wide range of triggers than non-asthmatics, leading to excessive narrowing with consequent reduced airflow and symptomatic wheezing and dyspnea (Barnes et al., 2016 \& Kasper et al., 2015). In Alexandria Main University Hospital, the statistical record showed that, 2324 patients were admitted to the Medical Chest Disease Department during the year of 2017, and patients with bronchial asthma represented about one fifth and half of the total admission rate (Statistical records of Alexandria Main University Hospital., 2019).

Asthma is characterized by variable symptoms of wheezes, shortness of breath, chest tightness, fatigue and/or cough, in addition to variable limitation of the expiratory airflow. It is characterized by an unpredictable course, attacks that often occur at night and may last for few minutes to several hours. Between attacks patients may be asymptomatic with normal or near normal pulmonary function, depending on severity of disease (Baiardini et al., 2015). Dyspnea is a primary symptom of asthma as it is the clinical manifestation of episodic bronchospasm; it is generally reported by patients to indicate an unpleasant stimulus often accompanied by multiple symptoms as chest tightness, difficulty of inspiration, unrewarded inspiration, and difficult in expiration process. It is considered one of the most disabling symptoms in patients with bronchial asthma and obviously the trigger point of physical inactivity as performing activities of daily living (ADL) that results in a higher ventilatory demand and less ventilatory efficiency. As a result of increased dyspnea, patients adapt themselves by reducing the amount of all ADL performed, with a consequent lower standard of quality of life (Antonelli et al., 2013, Barusso \& Santos, 2014 Pietiläinen et al., 2010 \& William et al., 2016 ).

The second important symptom for patients with asthma after dyspnea is fatigue, which is considered one of the most prominent disabling symptoms; it is also strongly associated with a decline in daily functional activity and a significant impairment of quality of life. Although pharmacologic treatment of asthma is of proven benefit, it doesn't help all patients as the side effects of pharmacotherapy can intensify respiratory effort, increase respiratory muscle weakness and add to the cost of management Therefore, it is recommended to examine other modalities for effective management of symptoms 
such as dyspnea and fatigue to improve individual quality of life (El-Saadawy, 2013).

One of the important non-pharmacological modalities in the traditional Chinese medicine (TCM) is Acupressure, which was originated in ancient China and showed greater therapeutic potentials against various disease conditions. It is considered as a type of acupuncture; both acupressure and acupuncture are based on the same basic principles. However, rather than using needles in case of acupuncture, acupoints are typically triggered using pressure from fingers, hands, elbows or, in some cases, by devices in acupressure (Mehtaa et al., 2017).

In accordance with traditional Chinese medicine, health is maintained by equilibrium between yin and yang and the free flow of energy, commonly known as qi or chi. any disturbance to the equilibrium of qi, can cause disease (Mehtaa et al., 2017) Qi passes through pathways called meridians. There are 12 main meridians and 8 extra in our body. Organs in our body are categorized either to yin (zang ) or yang (fu). Yin organs; are the heart, liver, spleen, lungs and kidneys, while the Yang organs involve gallbladder, stomach, small intestine, large intestine, and urinary bladder (Mehtaa et al., 2017 \& Ngai \& Chan, 2016).

Acupressure corrects the disequilibrium between qi through channels and consequently treats the diseases. Re-equilibrium of qi attains therapeutic benefits by improving the physiological functions of body systems or Zang-fu organs. It has many mechanisms of action especially in pain management, while definitely in case of the respiratory problems; it is based on the basic principle of activation of acupoints across the meridians (Mehtaa et al., 2017 \& Ngai \& Chan., 2016).

The biochemical mechanism of acupressure involves the stimulation of acupoints that leads to complex neuro-hormonal responses. It involves the counteraction between hypothalamic-pituitaryadrenocortical axis that leads to cortisol overproduction and cause a relaxation response. Also, it modulates physiological response by increasing the transmission of endorphin and serotonin to the brain and to specific organs. Thus, the analgesic and sedative effect of endorphins facilitates patients ' normal respiratory function and improves the effectiveness of breathing movements, that translates into larger tidal volumes (El-Saadawy, 2013).

In addition to the muscle relaxant effect of endorphins, they may reduce shallow breathing; allow deeper breathing movements, resulting in higher tidal volumes. Both can explain why most respiratory patients feel calm, warm, and relaxed during and after acupressure sessions, and why most patients with report dyspnea relief after acupressure (Maa \& Hsu., 2013). Hence dyspnea and fatigue are the most disabling symptoms in asthma and the trigger point of physical inactivity with a consequent lower standard of quality of life, and acupressure leads to the reduction of asthma symptoms and ultimately enhances asthma control; this study aimed to evaluate the effect of acupressure sessions on dyspnea and fatigue among patients with bronchial asthma. In an attempt to offer dyspnea and fatigue control, nurses are increasingly offering acupressure as part of their focus on comprehensive patient care. For proper administration of acupressure, the nurses should; choose a comfortable, private environment that lends itself to deep relaxation, teach the patient breathing exercise, master the proper allocation methods of the selected acupoints, use prolonged finger pressure directly on the point for proper acupressure application (Maa \& Hsu., 2013).

Aim of the study is

To evaluate the effect of acupressure sessions on dyspnea and fatigue among patients with bronchial asthma

\section{Research Hypothesis:}

Patients with bronchial asthma who are enrolled in acupressure sessions will exhibit lower grade of dyspnea and fatigue than those who do not.

\section{Materials \& Method \\ Research design:}

A quasi experimental research design was utilized to fulfill the aim of the present study.

\section{Settings:}

The present study was carried out at the Chest Inpatient Unit of Alexandria Main University Hospital. Inpatient chest department has a capacity of thirty-seven beds for male patients, and twentysix beds for female patients. The chest department has a lab for pulmonary function test. This hospital offers nonpaid public services for all governorates in Egypt.

Subjects:

The study subjects comprised a convenience sample of sixty (60) adult patients with a diagnosis of bronchial asthma, were selected based on Epi info7 program that was used to estimate the sample size using the following parameters:

1. Total population over one year ago at the above-mentioned hospital was 260 patients, 2018- 2019.

2. Expected frequency $=50 \%$

3. Acceptable error $=10 \%$

4. Confidence coefficient $=95 \%$

5. Estimated sample size is $=60$ patients with bronchial asthma.

They were enrolled according to the following criteria: able to communicate, free from any other respiratory disorders such as; T.B, pneumonia or any other associated diseases that lead to fatigue as; anemia, heart disease, musculoskeletal or neurological disorders. 
The study subjects were recruited and assigned randomly and alternatively into two equal groups; 30 patients each, namely:

- Control group (I): were subjected only to routine hospital treatment only (Asthma medications) of the Chest Department only.

- Experimental group (II): were enrolled to acupressure sessions and routine hospital treatment schedule.

\section{Tools}

Tool I: Patients with bronchial asthma assessment.

This tool was developed after reviewing the related literature (Osborn et al., 2010) to obtain baseline data. It consisted of two parts as follows:

Part I: Patient's demographic Characteristics; as age, sex, educational level, marital status, occupation, and residence area.

Part II: Patient's Clinical Data: as medical history, duration of disease, smoking habits, names and dosages of all prescribed medications.

Tool II: Dyspnea assessment questionnaire. Dyspnea was evaluated by using the modified Medical Research Council (mMRC) questionnaire (Launois et al., 2012). The mMRC breathlessness scale ranges from grade 0 to 4 . It comprises five items containing statements about the impact of breathlessness on the individual. (Grade 0); "I only get breathless with strenuous exercise". (Grade 1); "I get short of breath when hurrying on level ground or walking up a slight hill". (Grade 2); "On level ground, I walk slower than people of the same age because of breathlessness, or I have to stop for breath when walking at my own pace on the level". (Grade 3); "I stop for breath after walking about 300 yards (300 feet) or after a few minutes on level ground". (Grade 4); "I am too breathless to leave the house, or I am breathless when dressing".

The scoring system assigned as the following; (zero) was given to grade zero, (one) was given to grade one, (two) was given to grade two, (Three) was given to grade three, and (four) was given to grade four of dyspnea.

Tool III: Fatigue assessment questionnaire. This tool was developed based on a review of related literature (Benjamin et al., 2010 \& Eldeeb., 2016) to assess fatigue severity. It consists of two parts as follows:

Part I: Visual Analog Fatigue Scale (VAFS): This scale was adapted from (Benjamin et al., 2010)' and developed to assess fatigue severity. It is a horizontal line, $10 \mathrm{~cm}$ in length, anchored at each end by word descriptors; left end "No fatigue" and right end "Very severe fatigue "The patient marks the point he / she had felt on the line representing his/her perception and current state. The VAFS score are determined by measuring in centimeter from the left-hand end of the line to the point that the patient marked. The measured values are illustrated as (0) "no fatigue"; (1-3cm) "mild fatigue", $(4-6 \mathrm{~cm})$ "moderate fatigue" and $(7-9 \mathrm{~cm})$ "severe fatigue", (10) "very severe fatigue".

Part II: Multidimensional Fatigue Scale: This scale was adopted from Eldeeb H scale (2016). It aims to assess fatigue related to 4 dimensions; physical, psychological, mental and social. It is assessed on a 4 point rating scale ranging from (0) $=$ "Never experience fatigue", (1) = "rarely experience fatigue", (2) = "Often experience fatigue" and (3) = "almost always experience fatigue" (severe and continuous).

The mean percentage of the total Multidimensional Fatigue Scale score is classified as follows:

- Less than $50 \%$ = Means that fatigue does not interfere totally with physical, mental, psychological and social dimensions of patients with bronchial asthma.

- $50 \%$ to less than $80 \%=$ Means that fatigue partially interferes with physical, mental, psychological and social dimensions of patients with bronchial asthma.

- $80 \%$ and More = Means that fatigue totally interferes physical, mental, psychological and social dimensions of patients with bronchial asthma.

\section{Method}

- An approval from the Ethical Research Committee, Faculty of Nursing, Alexandria University was obtained.

- An official letter from the Faculty of Nursing was submitted to the general director of Alexandria Main University Hospital and to the Head of the Chest Disease Department of the above mentioned hospital for obtaining permission to carry out the study after complete explanation of the study aim.

- Tool I was developed, tool II was adopted from (Launois et al., 2012), and tool III was adapted from (Benjamin et al., 2010 \& Eldeeb., 2016) and the three tools were translated to Arabic.

- Tools Validity: All tools were submitted to five experts in the field of Chest Diseases Medicine, and Medical Surgical Nursing for content and validity and the necessary modifications were incorporated accordingly. The tools contents validity of each item (CVI) was assigned using the following index (CVI> 0.79, the item was relevant, CVI between 0.70 and 0.79 , the item was needed revision, while $\mathrm{CVI}<0.70$ the item was eliminated. CVI was determined by the number of experts giving a rating of relevant for each item divided by the total number of experts.

- The reliability of tools was tested by means of Cronbach's alpha. Reliability coefficient for tool I was (0.804), tool II was (0.907), and tool III was (0.904), which denotes high reliability.

- A pilot study was initially carried out prior to the actual data collection phase on five patients from 
the experimental study group to check clarity, feasibility and applicability of tools and identifying obstacles that may be encountered during the data collection process, and to determine the time needed to fill in the assessment tools, accordingly, the required modifications were done.

- Data collection started at the beginning of November 2018 and ended by April 2019.

The study was carried out through four phases Assessment Phase

- An initial assessment was carried out individually for every patient in both the control and experimental groups after careful listening and documenting history taking grading of dyspnea and fatigue severity using tool I, II, and III.

- The assessment session took from 30-60 minutes on individual basis.

- Subsequent assessment was carried out after 4 weeks thereafter in order to evaluate patient's progress for the both control and experimental groups.

Planning phase: A special training of in the field of acupressure was obtained by the study investigator at the Faculty of Physical Education, Alexandria University.

Implementation phase (Acupressure sessions for the experimental group):

- A comfortable, private environment conducive to deep relaxation was maintained prior to and throughout acupressure sessions.

- Patients were advised to wear comfortable clothes since tight collars, belts, pants, or shoes can obstruct circulation.

- Patient was informed not to eat at least 2 hours before the sessions; as practicing acupressure within a full stomach can inhibit the blood flow and may cause nausea.

- Patient was informed to avoid iced drinks (especially during the winter months), since extreme cold generally weakens the body's system and can counteract the benefits of acupressure.

- Deep breathing exercises were taught immediately before the session (take deep breathing from nose while counting four, hold breathing while counting two, exhale from mouth through pursed lips while counting four). Deep breathing helps to release any pain or tension from the acupressure points and encourages healing energy to flow through the whole body.

- Investigator's hands were kept warm by friction against each other, and nails short to prevent any patient's discomfort.

- The yin (lungs ; LU) meridians and the yang (large intestine or colon;LI) meridians were stimulated by making massage to both hands starting from shoulders toward the fingers from the inner surface of the arms and from the fingers toward the shoulder from the outer surface of the arms, twice.

- Accurate allocation of acuopoints was determined by use thumb or index on the allocated points. The middle finger is the longest and the most powerful finger therefore; the best suited for applying self-acupressure.

- Pressure was applied using finger massage in a slow, rhythmic manner with circular anti clockwise at five acupoints; (LU1, LU7, LU9, LI 4, and LI11), to stimulate the flow of energy and correct any imbalances. Figure.

\section{- (LU; lungs)}

\section{(LI ;large intestine or colon)}

Fig (1): Acupressure points used in this study Acupressure was carried out twice daily for two consecutive weeks; duration of each session for

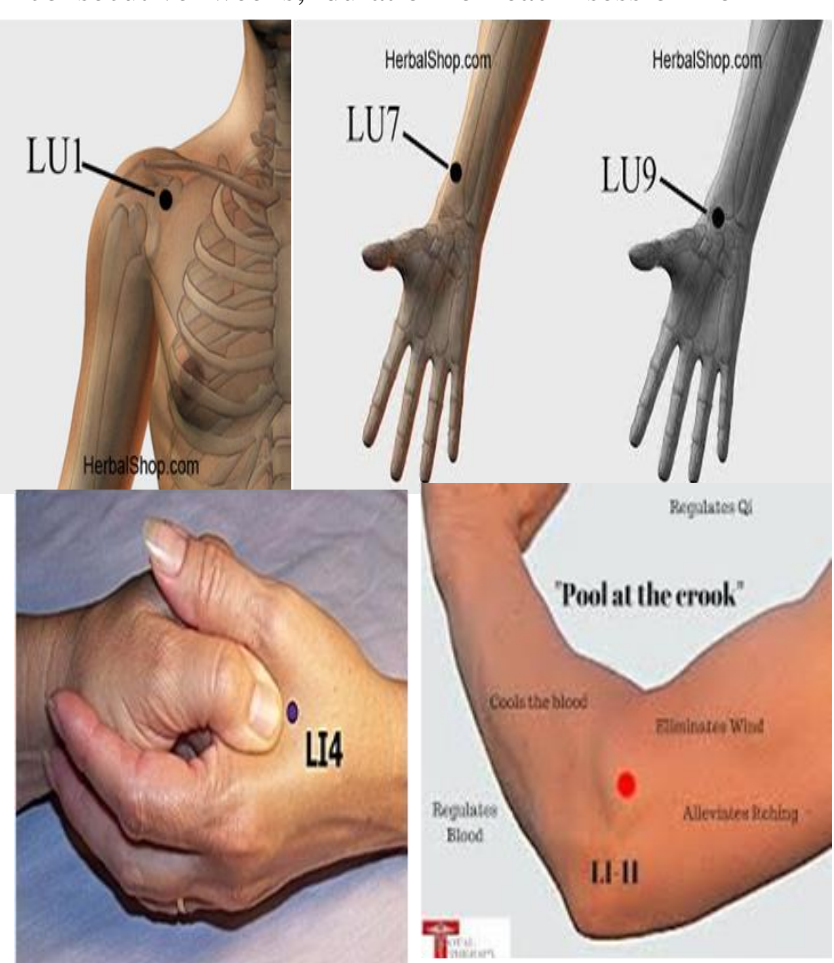

every patient was about $30-35$ minutes.

\section{Evaluation phase}

- Every Patient in the control and experimental group was re-evaluated after two weeks using tools II, and III.

- Comparisons between the findings of both groups were carried out using appropriate statistical analysis in order to determine the effect of acupressure sessions on dyspnea and fatigue among patients with bronchial asthma.

\section{Ethical consideration}

- A written informed consent from patients to participate in the study was obtained before data collection and after explanation of the aim of the study.

- Privacy of the study participants was asserted.

- Confidentiality of the collected data was assured.

- Participants' voluntary participation and their right to withdraw from the study at any time were emphasized. 


\section{Statistical analysis}

Data were processed and analyzed using PC with statistical package for social science (SPSS ver. 23)

Cronbach's alpha reliability test was used to measure the reliability of all tools. Its maximum value is $(\alpha=1.0)$ and the minimum accepted value is $(\alpha=0.7)$; below this level the tool would be unreliable Numbers and percentages from total were used to describe and summarize the demographic data. Comparisons within and between both groups were carried out using ChiSquare $(\chi 2)$ and Fisher's exact (FET) tests for grade of dyspnea, and fatigue severity. The level of significance decided for this study was $\mathrm{P}$ equal to or less than 0.05 (John., 2016).

\section{Results}

Table (1): Distribution of patients with bronchial asthma of both control and experimental groups according to demographic characteristics.

\begin{tabular}{|c|c|c|c|c|c|c|c|}
\hline \multirow{3}{*}{ Demographic characteristics } & \multicolumn{4}{|c|}{$(\mathrm{N}=60)$} & \multirow{2}{*}{\multicolumn{2}{|c|}{ Total }} & \multirow{3}{*}{$\begin{array}{c}\text { Significance } \\
\text { Test }\end{array}$} \\
\hline & \multicolumn{2}{|c|}{ Control Group } & \multicolumn{2}{|c|}{$\begin{array}{l}\text { Experimental } \\
\text { Group }\end{array}$} & & & \\
\hline & $\mathbf{N}=\mathbf{3 0}$ & $\%$ & $\mathbf{N}=\mathbf{3 0}$ & $\%$ & $\mathrm{~N}=60$ & $\%$ & \\
\hline $\begin{array}{l}\text { Age (years) } \\
\mathbf{2 0}-<\mathbf{3 0} \\
\end{array}$ & 6 & 20.0 & 6 & 20.0 & 12 & 20.0 & \multirow{5}{*}{$\begin{array}{l}\square^{2}=0.622 \\
P=0.891\end{array}$} \\
\hline $30-<40$ & 4 & 13.4 & 6 & 20.0 & 10 & 16.5 & \\
\hline $40-<50$ & 10 & 33.3 & 8 & 26.7 & 18 & 30.3 & \\
\hline $50-60$ & 10 & 33.3 & 10 & 33.3 & 20 & 33.2 & \\
\hline Total & 30 & 100 & 30 & 100 & 60 & 100 & \\
\hline $\mathrm{X} \pm \mathrm{SD}$ & \multicolumn{2}{|c|}{$39.60 \pm 12.287$} & \multicolumn{2}{|c|}{$43.40 \pm 14.202$} & & & \\
\hline $\begin{array}{l}\text { Sex } \\
\text { Male } \\
\end{array}$ & 2 & 6.7 & 1 & 3.3 & 3 & 5.0 & \multirow{3}{*}{$\begin{array}{l}\square^{2}=0.351 \\
\mathrm{P}=0.554\end{array}$} \\
\hline Female & 28 & 93.3 & 29 & 96.7 & 57 & 95.0 & \\
\hline Total & 30 & 100 & 30 & 100 & 60 & 100 & \\
\hline $\begin{array}{l}\text { Level of education } \\
\text { Illiterate }\end{array}$ & 11 & 36.7 & 13 & 43.3 & 24 & 40.0 & \multirow{6}{*}{$\begin{array}{c}\mathrm{FET}= \\
15.300 \\
\mathrm{P}=0.009 *\end{array}$} \\
\hline Read\& write & 7 & 23.3 & 5 & 16.7 & 12 & 20.0 & \\
\hline Primary +Preparatory & 9 & 30.0 & 0 & 0 & 9 & 15.0 & \\
\hline Secondary & 0 & 0 & 2 & 6.7 & 2 & 3.3 & \\
\hline $\begin{array}{l}\text { Education of the average, above } \\
\text { the average and higher } \\
\text { education }\end{array}$ & 3 & 10 & 10 & 33.3 & 13 & 21.7 & \\
\hline Total & 30 & 100 & 30 & 100 & 60 & 100 & \\
\hline $\begin{array}{l}\text { Marital status } \\
\text { Single }\end{array}$ & 4 & 13.3 & 6 & 20.0 & 10 & 16.6 & \multirow{5}{*}{$\begin{array}{c}\mathrm{FET}=5.427 \\
\mathrm{P}=0.143\end{array}$} \\
\hline Married & 19 & 63.4 & 18 & 60.0 & 37 & 61.7 & \\
\hline Divorced & 3 & 10.0 & 6 & 20.0 & 9 & 15.0 & \\
\hline Widow & 4 & 13.3 & 0 & 0 & 4 & 6.7 & \\
\hline Total & 30 & 100 & 30 & 100 & 60 & 100 & \\
\hline $\begin{array}{c}\text { Residence } \\
\text { Urban }\end{array}$ & 25 & 83.3 & 18 & 60.0 & 40 & 66.7 & \multirow{3}{*}{$\begin{array}{l}\square^{2}=1.200 \\
\mathrm{P}=0.174\end{array}$} \\
\hline Rural & 5 & 16.7 & 12 & 40.0 & 20 & 33.3 & \\
\hline Total & 30 & 100 & 30 & 100 & 60 & 100 & \\
\hline $\begin{array}{l}\text { Occupation } \\
\text { Not working } \\
\end{array}$ & 0 & 0 & 0 & 0 & 0 & 0 & \multirow{6}{*}{$\begin{array}{c}\mathrm{FET}=2.080 \\
\mathrm{P}=0.556\end{array}$} \\
\hline Clerical work & 0 & 0 & 1 & 3.3 & 1 & 1.7 & \\
\hline Manual work & 4 & 13.4 & 5 & 16.7 & 9 & 15.0 & \\
\hline Retired & 0 & 0 & 0 & 0 & 0 & 0 & \\
\hline Housewife & 26 & 86.6 & 24 & 80.0 & 50 & 83.3 & \\
\hline Total & 30 & 100 & 30 & 100 & 60 & 100 & \\
\hline
\end{tabular}

Chi-Square ( $\left.\chi^{2}\right)$ and Fisher's exact (FET)

"Significant difference at $P$ level $\leq 0.05$.

SD: standard deviation 
Table (2): Frequency distribution of patients with bronchial asthma of the both control and experimental groups according to clinical data.

\begin{tabular}{|c|c|c|c|c|c|c|c|}
\hline \multirow{3}{*}{ Clinical data } & \multicolumn{4}{|c|}{$(\mathrm{N}=60)$} & \multirow{2}{*}{\multicolumn{2}{|c|}{ Total }} & \multirow{3}{*}{$\begin{array}{c}\text { Significance } \\
\text { Test }\end{array}$} \\
\hline & \multicolumn{2}{|c|}{ Control Group } & \multicolumn{2}{|c|}{ Experimental Group } & & & \\
\hline & $\mathbf{N}=\mathbf{3 0}$ & $\%$ & $\mathbf{N}=\mathbf{3 0}$ & $\%$ & $\mathrm{~N}=60$ & $\%$ & \\
\hline $\begin{array}{l}\text { Presence of other health } \\
\text { problems } \\
\text { No }\end{array}$ & 29 & 96.7 & 24 & 80.0 & 53 & 88.3 & \multirow{6}{*}{$\begin{array}{c}\text { FET }=4.472 \\
\mathrm{P}=0.346\end{array}$} \\
\hline Diabetes & 0 & 0 & 1 & 3.4 & 1 & 1.7 & \\
\hline Hypertension & 1 & 3.3 & 3 & 10 & 4 & 6.6 & \\
\hline Liver \& kidney diseases & 0 & 0 & 1 & 3.3 & 1 & 1.7 & \\
\hline Cancer & 0 & 0 & 1 & 3.3 & 1 & 1.7 & \\
\hline Total & 30 & 100 & 30 & 100 & 60 & 100 & \\
\hline $\begin{array}{l}\text { Presence of other } \\
\text { respiratory diseases } \\
\text { No }\end{array}$ & 20 & 66.7 & 18 & 60.0 & 38 & 63.3 & \multirow{3}{*}{$\begin{array}{l}\square^{2}=0.287 \\
\mathrm{P}=0.592\end{array}$} \\
\hline Sinusitis & 10 & 33.3 & 12 & 40.0 & 22 & 36.7 & \\
\hline Total & 30 & 100 & 30 & 100 & 60 & 100 & \\
\hline $\begin{array}{l}\text { Family history of any } \\
\text { respiratory diseases } \\
\text { No }\end{array}$ & 21 & 70.0 & 16 & 53.3 & 37 & 61.7 & \multirow{4}{*}{$\begin{array}{l}\square^{2}=11.231 \\
P=0.004^{*}\end{array}$} \\
\hline Bronchial asthma & 4 & 13.3 & 14 & 46.7 & 18 & 30.0 & \\
\hline Sinusitis & 5 & 16.7 & 0 & 0 & 5 & 8.3 & \\
\hline Total & 30 & 100 & 30 & 100 & 60 & 100 & \\
\hline $\begin{array}{c}\text { Smoking habits } \\
\text { Nonsmoker }\end{array}$ & 8 & 26.7 & 11 & 36.7 & 19 & 31.6 & \multirow{5}{*}{$\begin{array}{c}\text { FET }=2.704 \\
P=0.439\end{array}$} \\
\hline Smoker & 1 & 3.3 & 0 & 0 & 1 & 1.7 & \\
\hline Quitter & 0 & 0 & 1 & 3.3 & 1 & 1.7 & \\
\hline Second-hand smoker & 21 & 70.0 & 18 & 60.0 & 39 & 65.0 & \\
\hline Total & 30 & 100 & 30 & 100 & 60 & 100 & \\
\hline
\end{tabular}

Chi-Square ( $\chi 2)$ and Fisher's exact (FET)

* Significant difference at $P$ level $\leq 0.05$.

Table (3): Frequency distribution of patients of both the control and experimental groups according to current disease history.

\begin{tabular}{|c|c|c|c|c|c|c|c|}
\hline \multirow{3}{*}{ Current disease history } & \multicolumn{4}{|c|}{$(\mathrm{N}=60)$} & \multirow{2}{*}{\multicolumn{2}{|c|}{ Total }} & \multirow{3}{*}{$\begin{array}{c}\text { Significance } \\
\text { Test }\end{array}$} \\
\hline & \multicolumn{2}{|c|}{$\begin{array}{l}\text { Control } \\
\text { Group }\end{array}$} & \multicolumn{2}{|c|}{$\begin{array}{c}\text { Experimental } \\
\text { Group }\end{array}$} & & & \\
\hline & $\mathbf{N}=\mathbf{3 0}$ & $\%$ & $\mathbf{N}=30$ & $\%$ & $\mathrm{~N}=60$ & $\%$ & \\
\hline $\begin{array}{l}\text { Age of starting bronchial asthma } \\
\text { (years) } \\
\text { less than } 10\end{array}$ & 2 & 6.6 & 13 & 43.4 & 15 & 25.0 & \multirow{5}{*}{$\begin{array}{l}\square^{2}=1.854 \\
\mathrm{P}=0.008 *\end{array}$} \\
\hline $10->3$ & 12 & 40.0 & 10 & 33.3 & 22 & 36.7 & \\
\hline $30->5$ & 8 & 26.7 & 3 & 10.0 & 11 & 18.3 & \\
\hline More than 50 & 8 & 26.7 & 4 & 13.3 & 12 & 20.0 & \\
\hline Total & 30 & 100 & 30 & 100 & 60 & 100 & \\
\hline $\begin{array}{l}\text { Symptoms felt when patient discover } \\
\text { bronchial asthma } \\
\text { Dyspnea }\end{array}$ & 28 & 93.3 & 24 & 80.0 & 51 & 85.0 & \multirow{7}{*}{$\begin{array}{l}\mathrm{FET}=19.74 \\
2 \\
\mathrm{P}=0.001 *\end{array}$} \\
\hline Productive cough & 27 & 90.0 & 20 & 66.7 & 48 & 80.0 & \\
\hline Fatigue most of the time & 24 & 80.0 & 6 & 20.0 & 30 & 50.0 & \\
\hline Chest wheezing & 5 & 16.7 & 4 & 13.3 & 9 & 15.0 & \\
\hline Chest tightness & 1 & 3.3 & 9 & 30.0 & 10 & 16.7 & \\
\hline Cyanosis & 0 & 0 & 3 & 10.0 & 3 & 5.0 & \\
\hline Total & & & & & & & \\
\hline
\end{tabular}




\begin{tabular}{|c|c|c|c|c|c|c|c|}
\hline \multirow{3}{*}{ Current disease history } & \multicolumn{4}{|c|}{$(\mathrm{N}=60)$} & \multirow{2}{*}{\multicolumn{2}{|c|}{ Total }} & \multirow{3}{*}{$\begin{array}{l}\text { Significance } \\
\text { Test }\end{array}$} \\
\hline & \multicolumn{2}{|c|}{$\begin{array}{l}\text { Control } \\
\text { Group }\end{array}$} & \multicolumn{2}{|c|}{$\begin{array}{c}\text { Experimental } \\
\text { Group }\end{array}$} & & & \\
\hline & $\mathbf{N}=\mathbf{3 0}$ & $\%$ & $\mathbf{N}=\mathbf{3 0}$ & $\%$ & $\mathrm{~N}=60$ & $\%$ & \\
\hline $\begin{array}{l}\text { Treatment taken since discovery of the } \\
\text { disease } \\
\text { Don't know }\end{array}$ & 16 & 53.3 & 19 & 63.3 & 35 & 58.3 & \multirow{6}{*}{$\begin{array}{c}\mathrm{FET}=5.927 \\
\mathrm{P}=0.204\end{array}$} \\
\hline Bronchodilators & 11 & 36.7 & 8 & 26.7 & 19 & 31.7 & \\
\hline Mucolytic or expectorants & 13 & 43.3 & 5 & 16.7 & 18 & 30.0 & \\
\hline Corticosteroids & 10 & 33.3 & 10 & 33.3 & 20 & 33.3 & \\
\hline Antibiotics & 0 & 0 & 2 & 6.7 & 2 & 3.3 & \\
\hline \multicolumn{7}{|l|}{ Total } & \\
\hline $\begin{array}{l}\text { Current Medications } \\
\text { Don't know }\end{array}$ & 8 & 26.7 & 15 & 50.0 & 23 & 38.3 & \multirow{6}{*}{$\begin{aligned} \mathrm{FET} & =4.558 \\
\mathrm{P} & =0.335\end{aligned}$} \\
\hline Bronchodilators & 20 & 66.7 & 18 & 60.0 & 34 & 56.7 & \\
\hline Mucolytic or expectorants & 16 & 53.3 & 14 & 46.7 & 34 & 56.7 & \\
\hline Corticosteroids & 9 & 30.0 & 5 & 16.7 & 14 & 23.3 & \\
\hline Antibiotics & 4 & 13.3 & 3 & 10.0 & 7 & 11.7 & \\
\hline \multicolumn{7}{|l|}{ Total } & \\
\hline $\begin{array}{l}\text { Previous hospitalization due to asthma } \\
\text { No }\end{array}$ & 13 & 43.3 & 7 & 23.3 & 20 & 33.3 & \multirow{5}{*}{$\begin{array}{l}\square^{2}=6.989 \\
\mathrm{P}=0.072\end{array}$} \\
\hline Less than 5 times & 11 & 36.7 & 18 & 60.0 & 29 & 48.4 & \\
\hline 5-10 times & 3 & 10.0 & 5 & 16.7 & 8 & 13.3 & \\
\hline More than 10 times & 3 & 10.0 & 0 & 0 & 3 & 5.0 & \\
\hline Total & 30 & 100 & 30 & 100 & 60 & 100 & \\
\hline
\end{tabular}

Chi-Square ( $\chi 2)$ and Fisher's exact (FET)

N.B: Some patients has more than one symptom, treatment taken since discovery of the disease, and current medications, therefore the number of each category exceeded the allocated sample size.

"Statistically Significant difference at P level $\leq 0.05$.

Table (4): Comparisons between the control and experimental groups regarding grade of dyspnea on initial assessment and two weeks post acupressure sessions.

\begin{tabular}{|c|c|c|c|c|c|c|c|c|c|c|}
\hline \multirow{4}{*}{ Grading of dyspnea } & \multicolumn{8}{|c|}{ Groups $(N=60)$} & \multirow{2}{*}{\multicolumn{2}{|c|}{ Test of Significance }} \\
\hline & \multicolumn{4}{|c|}{$\begin{array}{c}\text { Control } \\
\text { Group }(\mathbf{N}=\mathbf{3 0}) \\
\end{array}$} & \multicolumn{4}{|c|}{$\begin{array}{l}\text { Experimental } \\
\text { Group }(\mathrm{N}=30)\end{array}$} & & \\
\hline & \multicolumn{2}{|c|}{$\begin{array}{c}\text { Initial } \\
\text { assessment }\end{array}$} & \multicolumn{2}{|c|}{$\begin{array}{l}\text { After two } \\
\text { weeks }\end{array}$} & \multicolumn{2}{|c|}{$\begin{array}{c}\text { Initial } \\
\text { assessment }\end{array}$} & \multicolumn{2}{|c|}{$\begin{array}{c}\text { After two } \\
\text { weeks }\end{array}$} & \multirow[t]{2}{*}{ Pre } & \multirow[t]{2}{*}{ Post } \\
\hline & No & $\%$ & No & $\%$ & No & $\%$ & No & $\%$ & & \\
\hline Grade 0 & 0 & 0 & 0 & 0 & 0 & 0 & 0 & 0 & \multirow{7}{*}{$\begin{array}{c}\mathrm{FET}=20.418 \\
\mathrm{P}=0.023^{*}\end{array}$} & \multirow{7}{*}{$\begin{array}{c}\mathrm{FET}=13.784 \\
\mathrm{P}=0.000^{*}\end{array}$} \\
\hline Grade 1 & 0 & 0 & 0 & 0 & 1 & 3.3 & 7 & 23.3 & & \\
\hline Grade 2 & 7 & 23.3 & 8 & 26.7 & 4 & 13.3 & 10 & 33.3 & & \\
\hline Grade 3 & 13 & 43.3 & 12 & 40.0 & 14 & 46.7 & 9 & 30.0 & & \\
\hline Grade 4 & 10 & 33.4 & 10 & 33.3 & 11 & 36.7 & 4 & 13.4 & & \\
\hline Total & 30 & 100 & 30 & 100 & 30 & 100 & 30 & 100 & & \\
\hline Significance test & \multicolumn{4}{|c|}{$\begin{array}{c}\mathrm{FET}=1.047 \\
\mathrm{P}=0.902\end{array}$} & \multicolumn{4}{|c|}{$\begin{array}{c}\mathrm{FET}=16.060 \\
\mathrm{P}=0.013^{*}\end{array}$} & & \\
\hline
\end{tabular}


Table (5): Comparisons between patients in control and experimental group patients regarding Visual Analog Fatigue Scale grades on initial assessment and two weeks post acupressure sessions.

\begin{tabular}{|c|c|c|c|c|c|c|c|c|c|c|}
\hline \multirow{4}{*}{$\begin{array}{l}\text { Visual Analog Fatigue } \\
\text { Scale (VAFS) }\end{array}$} & \multicolumn{8}{|c|}{ Groups $(N=60)$} & \multirow{2}{*}{\multicolumn{2}{|c|}{ Test of Significance }} \\
\hline & \multicolumn{4}{|c|}{$\begin{array}{c}\text { Control } \\
\text { Group }(\mathrm{N}=\mathbf{3 0})\end{array}$} & \multicolumn{4}{|c|}{$\begin{array}{l}\text { Experimental } \\
\text { Group }(\mathrm{N}=30)\end{array}$} & & \\
\hline & \multicolumn{2}{|c|}{$\begin{array}{c}\text { Initial } \\
\text { assessment }\end{array}$} & \multicolumn{2}{|c|}{$\begin{array}{l}\text { After two } \\
\text { weeks }\end{array}$} & \multicolumn{2}{|c|}{$\begin{array}{c}\text { Initial } \\
\text { assessment }\end{array}$} & \multicolumn{2}{|c|}{$\begin{array}{c}\text { After two } \\
\text { weeks }\end{array}$} & \multirow[t]{2}{*}{ Pre } & \multirow[t]{2}{*}{ Post } \\
\hline & No & $\%$ & No & $\%$ & No & $\%$ & No & $\%$ & & \\
\hline No fatigue $(0)$ & 0 & 0 & 0 & 0 & 0 & 0 & 0 & 0 & \multirow{7}{*}{$\begin{array}{c}\mathrm{FET}=4.333 \\
\mathrm{P}=0.227\end{array}$} & \multirow{7}{*}{$\begin{array}{c}\mathrm{FET}=33.132 \\
\mathrm{P}=0.000^{*}\end{array}$} \\
\hline Mild fatigue $(1-3 \mathrm{~cm})$ & 0 & 0 & 0 & 0 & 3 & 10.0 & 18 & 60.0 & & \\
\hline Moderate fatigue $(4-6 \mathrm{~cm})$ & 5 & 16.7 & 4 & 13.3 & 7 & 23.3 & 7 & 23.3 & & \\
\hline Severe fatigue $(7-9 \mathrm{~cm})$ & 15 & 50.0 & 18 & 60.0 & 11 & 36.7 & 3 & 10.0 & & \\
\hline Very severe fatigue $(10 \mathrm{~cm})$ & 10 & 33.3 & 8 & 26.7 & 9 & 30.0 & 2 & 6.7 & & \\
\hline Total & 30 & 100 & 30 & 100 & 30 & 100 & 30 & 100 & & \\
\hline Test of Significance & \multicolumn{4}{|c|}{$\begin{array}{c}\mathrm{FET}=0.606 \\
\mathrm{P}=0.738\end{array}$} & \multicolumn{4}{|c|}{$\begin{array}{c}\text { FET }=19.816 \\
\mathrm{P}=0.000 *\end{array}$} & & \\
\hline
\end{tabular}

FET $=$ Fisher's exact test.

*Significant difference at $P$ level $\leq 0.05$ 
Table (6): Comparisons between control group regarding fatigue effect on physical, mental, psychological and social dimensions on initial assessment and two weeks post routine hospital treatment.

\begin{tabular}{|c|c|c|c|c|c|c|c|c|c|c|c|c|c|c|c|c|}
\hline \multirow{4}{*}{ Fatigue effect } & \multicolumn{16}{|c|}{ Fatigue dimensions } \\
\hline & \multicolumn{4}{|c|}{ Physical } & \multicolumn{4}{|c|}{ Mental } & \multicolumn{4}{|c|}{ Psychological } & \multicolumn{4}{|c|}{ Social } \\
\hline & \multicolumn{2}{|c|}{$\begin{array}{c}\text { Initial } \\
\text { assessment }\end{array}$} & \multicolumn{2}{|c|}{$\begin{array}{c}\text { Two weeks } \\
\text { After } \\
\end{array}$} & \multicolumn{2}{|c|}{$\begin{array}{c}\text { Initial } \\
\text { assessment }\end{array}$} & \multicolumn{2}{|c|}{$\begin{array}{c}\text { Two weeks } \\
\text { After } \\
\end{array}$} & \multicolumn{2}{|c|}{$\begin{array}{c}\text { Initial } \\
\text { assessment }\end{array}$} & \multicolumn{2}{|c|}{$\begin{array}{c}\text { Two weeks } \\
\text { after }\end{array}$} & \multicolumn{2}{|c|}{$\begin{array}{c}\text { Initial } \\
\text { assessment }\end{array}$} & \multicolumn{2}{|c|}{ Two weeks after } \\
\hline & No & $\%$ & No & $\%$ & No & $\%$ & No & $\%$ & No & $\%$ & No & $\%$ & No & $\%$ & No & $\%$ \\
\hline Totally interferes & 16 & 53.3 & 18 & 60.0 & 0 & 0.0 & 0 & 0.0 & 12 & 40.0 & 12 & 40.0 & 5 & 16.7 & 8 & 26.7 \\
\hline Partially interferes & 14 & 46.7 & 12 & 40.0 & 17 & 56.7 & 17 & 56.7 & 18 & 60.0 & 18 & 60.0 & 24 & 80.0 & 21 & 70.0 \\
\hline Not interfere & 0 & 0.0 & 0 & 0.0 & 13 & 43.3 & 13 & 43.3 & 0 & 0 & 0 & 0 & 1 & 3.3 & 1 & 3.3 \\
\hline Total & 30 & 100 & 30 & 100 & 30 & 100 & 30 & 100 & 30 & 100 & 30 & 100 & 30 & 100 & 30 & 100 \\
\hline Significance test & \multicolumn{4}{|c|}{$\begin{aligned} \square^{2} & =3.0853 \\
\mathrm{P} & =0.213\end{aligned}$} & \multicolumn{4}{|c|}{$\begin{array}{l}\square^{2}=- \\
P=-\end{array}$} & \multicolumn{4}{|c|}{$\begin{array}{l}\square^{2}=- \\
P=-\end{array}$} & \multicolumn{4}{|c|}{$\begin{array}{l}\square^{2}=12.226 \\
\mathrm{P}=0.016^{*}\end{array}$} \\
\hline
\end{tabular}

Table (7): Comparisons between experimental group regarding fatigue effect on physical, mental, psychological and social dimensions on initial assessment and two weeks after acupressure sessions.

\begin{tabular}{|c|c|c|c|c|c|c|c|c|c|c|c|c|c|c|c|c|}
\hline \multirow{4}{*}{ Fatigue effect } & \multicolumn{16}{|c|}{ Fatigue dimensions } \\
\hline & \multicolumn{4}{|c|}{ Physical } & \multicolumn{4}{|c|}{ Mental } & \multicolumn{4}{|c|}{ Psychological } & \multicolumn{4}{|c|}{ Social } \\
\hline & \multicolumn{2}{|c|}{$\begin{array}{c}\text { Initial } \\
\text { assessment }\end{array}$} & \multicolumn{2}{|c|}{$\begin{array}{c}\text { After two } \\
\text { weeks }\end{array}$} & \multicolumn{2}{|c|}{$\begin{array}{c}\text { Initial } \\
\text { assessment }\end{array}$} & \multicolumn{2}{|c|}{$\begin{array}{c}\text { Two weeks } \\
\text { After }\end{array}$} & \multicolumn{2}{|c|}{$\begin{array}{c}\text { Initial } \\
\text { assessment }\end{array}$} & \multicolumn{2}{|c|}{$\begin{array}{c}\text { Two weeks } \\
\text { After }\end{array}$} & \multicolumn{2}{|c|}{$\begin{array}{c}\text { Initial } \\
\text { assessment }\end{array}$} & \multicolumn{2}{|c|}{ Two weeks After } \\
\hline & No & $\%$ & No & $\%$ & No & $\%$ & No & $\%$ & No & $\%$ & No & $\%$ & No & $\%$ & No & $\%$ \\
\hline Totally interferes & 11 & 36.7 & 4 & 13.4 & 0 & 0.0 & 0 & 0.0 & 5 & 16.7 & 2 & 6.7 & 5 & 16.7 & 2 & .6 .7 \\
\hline Partially interferes & 17 & 56.7 & 24 & 80.0 & 16 & 53.3 & 5 & 16.7 & 25 & 83.3 & 26 & 86.6 & 22 & 73.3 & 18 & 60.0 \\
\hline Not interfere & 2 & 6.6 & 2 & 6.6 & 14 & 46.7 & 25 & 83.3 & 0 & 0.0 & 2 & 6.7 & 3 & 10.0 & 10 & 33.3 \\
\hline Total & 30 & 100 & 30 & 100 & 30 & 100 & 30 & 100 & 30 & 100 & 30 & 100 & 30 & 100 & 30 & 100 \\
\hline Significance test & & $\begin{array}{l}\square^{2}= \\
\mathrm{P}=\end{array}$ & $\begin{array}{l}.533 \\
00 *\end{array}$ & & & $\begin{array}{l}\square^{2}= \\
P=(\end{array}$ & $\begin{array}{l}473 \\
24 *\end{array}$ & & & $\begin{array}{l}\square^{2}=2 \\
\mathrm{P}=0\end{array}$ & $\begin{array}{l}322 \\
00^{*}\end{array}$ & & & & $\begin{array}{l}=10.8 \\
=0.02\end{array}$ & \\
\hline
\end{tabular}


Table (8): Comparisons between control and experimental groups regarding total mean percentage of Fatigue effect on initial assessment and two weeks post acupressure sessions.

\begin{tabular}{|c|c|c|c|c|c|c|c|c|c|c|}
\hline \multirow{4}{*}{ Fatigue effect } & \multicolumn{8}{|c|}{ Groups $(N=60)$} & \multirow{2}{*}{\multicolumn{2}{|c|}{ Significance test }} \\
\hline & \multicolumn{4}{|c|}{$\begin{array}{c}\text { Control } \\
\text { Group }(\mathrm{N}=\mathbf{3 0})\end{array}$} & \multicolumn{4}{|c|}{$\begin{array}{l}\text { Experimental } \\
\text { Group }(\mathrm{N}=30)\end{array}$} & & \\
\hline & \multicolumn{2}{|c|}{$\begin{array}{c}\text { Initial } \\
\text { assessmen } \\
\mathbf{t} \\
\end{array}$} & \multicolumn{2}{|c|}{$\begin{array}{c}\text { Two weeks } \\
\text { After }\end{array}$} & \multicolumn{2}{|c|}{$\begin{array}{c}\text { Initial } \\
\text { assessment }\end{array}$} & \multicolumn{2}{|c|}{$\begin{array}{c}\text { Two weeks } \\
\text { After }\end{array}$} & \multirow[t]{2}{*}{ Pre } & \multirow[t]{2}{*}{ Post } \\
\hline & No & $\%$ & No & $\%$ & No & $\%$ & No & $\%$ & & \\
\hline $\begin{array}{l}\text { - Fatigue totally interferes with } \\
\text { physical, mental, psychological } \\
\text { and social aspects of patients } \\
\text { with bronchial asthma. }\end{array}$ & 16 & 53.3 & 13 & 43.3 & 18 & 60.0 & 5 & 16.7 & \multirow{4}{*}{$\begin{array}{c}\chi 2=0 \\
271 \\
P=0.602\end{array}$} & \multirow{5}{*}{$\begin{array}{l}\chi 2=8.799 \\
P=0.012 *\end{array}$} \\
\hline $\begin{array}{l}\text { - Fatigue partially interferes with } \\
\text { physical, mental, psychological } \\
\text { and social aspects of patients } \\
\text { with bronchial asthma. }\end{array}$ & 14 & 46.7 & 17 & 56.7 & 12 & 40.0 & 20 & 66.6 & & \\
\hline $\begin{array}{l}\text { - Fatigue doesn't interfere with } \\
\text { physical, mental, psychological } \\
\text { and social aspects of patients } \\
\text { with bronchial asthma. }\end{array}$ & 0 & 0 & 0 & 0 & 0 & 0 & 5 & 16.7 & & \\
\hline Total & 30 & 100 & 30 & 100 & 30 & 100 & 30 & 100 & & \\
\hline Significance test & \multicolumn{4}{|c|}{$\begin{array}{l}\chi 2=0.600 \\
P=0.438\end{array}$} & \multicolumn{4}{|c|}{$\begin{array}{l}\chi^{2}=14.347 \\
P=0.000 *\end{array}$} & & \\
\hline
\end{tabular}

$\chi^{2}=$ Chi-Square.

*Significant difference at $P$ level $\leq 0.05$.

Table (1): Showed that less than half of the sample $33.2 \%$ were among the age group of (50 60 years old), distributed equally between both groups as $33.3 \%$ in each group without statistically significant difference. The highest percent of patients $95 \%$ were females, and more than one thirds of them $40 \%$ among both groups were illiterate. More than two-thirds $83.3 \%$ of the control patients and around two-third $66.7 \%$ of the experimental were coming from urban area. Around two thirds of both control and experimental patients were married; and it is clear that the majority of patients were housewives $83.3 \%$.

Table (2): Showed that the majority of patients among both control and experimental groups $(96.7 \%, 80 \%)$ respectively had no associated diseases. Two thirds $66.7 \%$ of the control group patients hadn't other respiratory diseases, while $60 \%$ of the experimental hadn't other respiratory diseases, and more than one-third $40 \%$ had sinusitis. No family history was detected among the control and experimental groups $(70 \%, 53.3 \%)$ respectively. Most of the patients were secondhand smokers (70\% and 60\%) among the control and the experimental groups, respectively.

Table (3): Illustrated that more than one third $40 \%$ of the control group subjects, their disease onset were by the age of ten to less than thirty, while more than one third $43.4 \%$ of the experimental group had their disease onset by the age of less than ten years. Dyspnea was the main symptom encountered among both groups representing (93.3\% and $80 \%)$ of the control and experimental groups patients respectively. The majority of patients were found to use bronchodilators. It was observed that about morethan one third $43.3 \%$ of the control group patients had no previous hospitalization. On the other hand, around two-thirds $60 \%$ the patients in the experimental group were hospitalized less than five times because of bronchial asthma attacks.

Table (4): Revealed that dyspnea (grade 3) was the most frequently encountered grade among control groups on the initial assessment while after two weeks post routine hospital treatment this percent was decreased (43.3\%, and 40.0\%) respectively. In relation to the experimental group, $46.7 \%$ of patients complained of dyspnea (grade 3 ) on the initial assessment, while after two weeks post acupressure sessions, this percent was decreased to $33.3 \%$ of patients who had dyspnea (grade 2). Significant differences were observed between the control and experimental subjects with $\left(\mathrm{FET}=16.606, \mathrm{P}=0.013^{*}\right),(\mathrm{FET}=13.784, \mathrm{P}$ $=0.000 *)$ respectively.

Table (5): Illustrated that half of the patients in the control group i.e. $50 \%$ complained of severe fatigue on the initial assessment, while more than half of them $60 \%$ showed no decrease in the severity of fatigue level after two weeks from the routine hospital treatment schedule. In contrast, $36 \%$ of the patients in the experimental group had severe fatigue, while after acupressure sessions, this percent was decreased, as more than half of the patients $60 \%$ had mild fatigue and $10 \%$ only 
had severe fatigue. High significant differences were observed within the experimental group between the pre- and post-assessment. Also, high significant differences were observed between the control and experimental groups in both the initial assessment and after two weeks with $(\mathrm{P}=0.000$ *, $\mathrm{P}=0.000 *$ ) respectively.

Table (6): Showed that fatigue interferes with patient's physical, mental, psychological and social dimensions, without significant difference except in relation to the social dimension there was significant difference with $\left(\mathrm{P}=0.016^{*}\right)$.

Table (7): Showed that fatigue experienced by most of the patients partially interferes with their physical, psychological and social dimensions pre and post application of acupressure with significant difference $\left(\mathrm{P}=0.000 *, \mathrm{P}=0.000^{*}, \mathrm{P}=\right.$ $\left.0.028^{*}\right)$ respectively. On the other hand, the majority of them their fatigue partially interferes with their mental dimension pre the application of acupressure while, post the application of acupressure the majority of patients their fatigue does not interfere with their mental dimension with significant difference $\left(\mathrm{P}=0.024^{*}\right)$.

Table (8): Illustrated that fatigue partially interferes with physical, mental, psychological and social aspects of patients with bronchial asthma on initial assessment and after two weeks post routine hospital treatment schedule and acupressure sessions, with superiority to post the application of acupressure. The difference was highly statistically significant within the experimental group, and also between the control and experimental subjects post the application of acupressure $(\mathrm{P}=0.000 *, \mathrm{p}=0.012 *)$ respectively.

\section{Discussion}

In recent years, the demand for complementary therapies among chronic disease patients has gained considerable feedback, in particular for acupressure. It is an inexpensive and safe treatment option without side effects, which in part leads to its popularity among patients with bronchial asthma. (Maa \& Hsu, 2013).

Concerning demographic data, the present study demonstrated that the majority of patients were in the late adult age. This may be explained by age-related changes in lung physiology and morphology including marked decrease in elastic recoil, increased chest wall rigidity, and decreased respiratory muscle strength. Structural changes of the aging lung may worsen the physiologic function among patients with bronchial asthma, in addition the induced changes in the immune system due to aging; increased vulnerability to pathogens and effect of inflammation on the airway, all these factors leading to variability in how asthma manifests and patient responds to treatment. This result was in congruent with the results of (Zein et al., 2015), who mentioned that the probability of severe asthma increased frequently with the aged between 51 and 57 years old (Budde \& Skloot, 2018)

The disparity in asthma between men and women is mostly due to female's hormones as estrogen variations which cause asthma to become exacerbations among women due to its antiinflammatory activity, that drop during menopause and in women with bronchial asthma compared to healthy women (Zein et al.,2015).

In relation to sex, more than three quarters of patients were females. This was supported by a study of (Pigantaro et al., 2017) who stated that asthma has a higher incidence in females and appears to have more asthma symptoms than men and to use more rescue medications, leading to a reduced quality of life. Also, (Greenblatt et al., 2017) reported that asthma was found to be significantly higher among females.

In relation to the level of education, it was observed that the highest percentage of patients in both groups were illiterate. This result was matching with the findings of (Koch, 2017) who studied the prevalence of asthma among adults in Germany and found that overall women and men with a low educational backgrounds more frequently reported to have had asthma more than those with a medium or high level of education. This finding was not in line with (Al-Mazam \& Mohamed, 2011) who reported that there was no association between education and bronchial asthma.

The present findings could be attributed to the fact that poor educational level is considered a significant factor for increasing the exposure to asthma triggers and asthma episodes. In addition to the setting of the study which was a public university governmental hospital that serves not only urban areas but also many rural areas and the majority of patients had low educational and economical standards .

The residence may give an idea about the circumstances in which the patients live. As the majority of the patients in the present study were living in urban areas. This was in accordance with (Rambabu et al., 2016) who stated that urban areas had higher prevalence of asthma compared to rural areas. While (Keet et al., 2017) who reported that residence is an important risk factor for asthma morbidity in rural and urban areas. This finding was attributed to that exposure to air pollution has adverse effects on human health, increasing the risk of respiratory diseases. Higher pollution of the environment due to industrial / manufacturing activities and the presence of state highways can cause air pollution from motor vehicle traffic and increase induction of asthma among people.

It was obvious that the majority of patients in the current study were housewives. This result was in 
harmony with (Tageldin et al., 2015) who illustrated that more than three-quarters of the patients in their study were housewives, and about one quarter were employed and consequently exposed to indoor and outdoor pollution This finding may also be due to that housewives mainly spend more than $90 \%$ of their time indoors, as reported by patients, making them exposed to indoor allergens including secondhand smoke, cooking, biomass burning in stoves, and cleaning activities that can stimulate an IgE response, which in turn causes health effects ranging from sneezing and coughing to increasing asthma exacerbation.

With reference to the presence of other respiratory diseases among patients with bronchial asthma, it was found that two-thirds of patients in the control group were not having any other respiratory diseases, with the remainder that sinusitis was reported. Also, more than-one third is having sinusitis. These results supported by (Shaw et al., 2015) who found that the prevalence of chronic sinusitis ranges from $45-50 \%$ in patients with severe asthma. Also (Brinke et al., 2012) reported that the majority of patients with severe asthma had sinusitis in the computed tomography (CT) findings. This finding may reflect a systemic inflammatory process of the respiratory mucosa. Eosinophil inflammation and local IgE production may occur in both.

As for Family history of respiratory diseases, no patients with a family history of respiratory disease were encountered among the majority of the both patients groups'; this is to some extent similar to (Cogswell., 2010) finding, who did not found strong associations between asthma and history of asthma in the mother versus the father. However, this is not in line with the study done by (Burake W et al., 2013) who varied a positive family history and asthma association.

As regards smoking habits, the majority of patients in both groups were secondhand smokers. No doubt, secondhand smoking increases the likelihood of experiencing respiratory problems and is associated with increased bronchial responsiveness (Jang et al., 2014). This result is sustained by (Kim et al., 2017) who illustrated that secondhand smoking showed positive relation with occurrence of asthma. Also, (Kim et al., 2018) reported that longer passive smoking was related to wheezing and exercise wheezing.

With reference to age when bronchial asthma started, the findings of the present study showed that more than one-third of the control group patients had their disease started at the age of ten to less than thirty, while more than one-third of the study group patients started their disease at the age of less than ten years old. This finding was matched with (Beavers et al., 2013) who reported that asthma onset before the age of sixteen was reported by the majority of adults with active asthma, followed by one third with onset at five to nine years of age.

With reference to symptoms felt when patient discover bronchial asthma, dyspnea was the main symptom encountered among both groups' of the patients, and this was in congruent with the result of (Lavietes, 2015) who concluded that selfreported dyspnea was the main symptom among patients with asthma . In addition, (Refaata \& Aref , 2014) argued that dyspnea was the most common chest symptom encountered in patients with acute asthma followed by wheezing .

Medications of different classes have been found to be useful in treating asthma and can be used in combination. The present study portrayed that the majority of patients among both groups used bronchodilators. Bronchodilators have played a main role in the treatment of asthma for a long time as they improve symptoms and lung functions primarily by relaxing airway smooth muscles. This result was in line with (Townsend et al., 2012) who indicated that the bronchodilators are the first line therapy to reverse airway obstruction during acute asthma exacerbations.

Concerning previous hospitalization as a result of bronchial asthma, it was found that about more than one third of the controls had no previous hospitalization. On the other hand, around twothirds of the experimental group were hospitalized less than five times because of asthma attacks. This finding was consistent with (Hasegawa et al., 2013) who stated that the majority of patients had twice times hospitalization, while the minority had three times hospitalization as a result of acute asthma exacerbations. This result may be related to that the majority of the patients had poor asthma control, and asthma exacerbations are frequent in patients with uncontrolled asthma That carries a significant burden of morbidity.

With reference to dyspnea grading, the present study portrayed that grade four was the most commonly encountered grade among control and experimental patients on the initial assessment and two weeks post routine hospital treatment and acupressure sessions. This grade was changed post acupressure session among the experimental patients only, as grade three was the most commonly encountered. These changes showed significant differences within the experimental group only, and between control and experimental subjects. These findings may be attributed to that acupressure produces muscle relaxation and promotes feeling of comfort as well, this relaxation also occurs for respiratory muscles. Moreover, respiratory muscle relaxation promotes comfort as well as anxiety and tension relief which might play a role of beneficial effect on dyspnea.

Furthermore, adrenocorticotropic hormone release may be stimulated by acupressure which causes the adrenal gland to release cortisol and produce 
anti-inflammatory effects that in turn decrease respiratory resistance. These results of the present study were congruent with (Trott \& Oei, 2019) who concluded that there was significant improvement in dyspnea in breathlessness severity in patients with advanced asthma following acupressure sessions. In this respect (El-Saadawy, 2013) ensured that the acupressure group had statistically significant improvement in relation to dyspnea respiratory rate and oxygen saturation than the controls.

Concerning fatigue severity, it was clear that severe fatigue was the most commonly fatigue severity category among control group patients on initial assessment and after two weeks post routine hospital treatment and acupressure sessions. Highly significant difference was observed within the experimental patients only and between the control and experimental groups' patients after application of acupressure. Furthermore, it was obvious that fatigue partially interferes with asthmatic patient's physical, mental, psychological and social aspects pre and post application of acupressure, with improvement post the application of acupressure. Pursed lips breathing, which is the starting point of acupressure technique, helps to conserve energy and promote daily activities with less effort and less shortness of breath by increasing effectiveness of breathing. In this context, (Tang et al., 2014) reported that acupressure with or without essential oils helps patients suffering from lung cancer undergoing chemotherapy, reduce cancer-related fatigue and increase activity level. Also, (Bastani et al., 2015) clarified that there were significant reductions of the mean fatigue score in the acupressure group compared to the placebo group immediately, two and four weeks after the intervention. (Vagharseyyedin et al., 2018) indicated that the mean scores of fatigue significantly decreased in the acupressure group than in the other group. Further, (El Fadawy et al., 2018) proved that acupressure has a significant improvement in the physical and psychological health status.

(El-Saadawy, 2013) had unsimilar findings when investigating the effect of acupressure on dyspnea and fatigue among patients with chronic obstructive pulmonary disease and revealed that there was no statistically significant improvement in relation to fatigue after application of acupressure. This difference could be interpreted in the light of the fact that chronic obstructive pulmonary disease is irreversible airway disease, while asthma is reversible.

Finally, the obtained results have put in evidence that acupressure has a positive effect in decreasing the grade of dyspnea, as well as fatigue severity. Nurses are become increasingly involved in providing complementary-alternative medicine (CAM) as part of their caring-healing focus and attempts to provide comfort measures, pain control and symptoms management for comprehensive patient care (Bastani et al., 2015).

\section{Conclusion}

From the findings of the present study, it can be concluded that:

- The therapeutic effects of acupressure sessions on the experimental group was were much better than that of control group in relation to dyspnea and fatigue severity, and the difference was statistically significant.

- Fatigue partially interferes with physical, mental, psychological and social aspects of patients with bronchial asthma, with superiority to post the application of acupressure. The difference was highly statistically significant within the experimental group, and between control and experimental groups post the application of acupressure

\section{Recommendations}

Based upon the findings of the study, the following recommendations are:

- Patients with bronchial asthma need teaching sessions on how to perform acupressure in the early course of the disease in order to decrease grading of dyspnea and restore energy .

- Additional studies may be needed using to explore different acupressure points to manage dyspnea, and fatigue among patients with bronchial asthma.

- Illustrated self-administered acupressure intervention booklets distributed to patients with bronchial asthma, could be of help.

- Development and implementation of acupressure training program for nurses, health care personnel and caregivers about acupressure are advocated.

\section{References}

- Al-Mazam A., \& Mohamed A., (2011): Risk factors of bronchial asthma in Bahrah, Saudi Arabia. J Family Community Med; 8(1): 33-9.

- Antonelli A., Crimi E., Gobbi A., Torchio R., \& Gulotta C., (2013): Mechanical correlates of dyspnea in bronchial asthma. Physiol Rep; 11(7):100-66.

- Baiardini I., Sicuro F., Balbi F., \& Canonica G., (2015): Psychological aspects in asthma: do psychological factors affect asthma management. Asthma Research and Practice; 1(7):25-31.

- Barnes P., Rodger L., \& Thomson N., (2016): Asthma basic Mechanisms and Clinical Management. $3^{\text {th }}$ ed. New York. Elsevier; 942.

- Barusso M., \& Santos J., (2014): Limitation of activities of daily living and quality of life based on COPD combined classification. Resp care; 10(4):187-96 
- Bastani F., Sobhani M., Emamzadeh \& H., (2015): Effect of acupressure on fatigue in women with multiple sclerosis. Glob J Health Sci 26;7(4):375-81.

- Beavers S., Chatterjee A., \& Moorman J., (2013): Age at asthma onset and subsequent asthma outcomes among adults with active asthma. Respir Med; 107(12): 1829-36.

- Benjamin Y., Tseng J., \& Patricia M., (2010): Department of Physical Therapy, and Rehabilitation Science, The Reliability, Responsiveness, and Validity of the Visual Analog Fatigue Scale to Measure Exertion Fatigue in People with Chronic Stroke: A Preliminary Study .Stroke Research and Treatment, 41(4) :7 -25.

- Brinke A., Grootendorst D., Schmidt J., Bruïne F., Buchem M., Sterk P., Rabe K., \& Bel E., (2012): Chronic sinusitis in severe asthma is related to sputum eosinophilia. J Allergy Clin Immunol; 109(4):621-6. 189.

- Budde J., Skloot G., (2018): Is aging a comorbidity of asthma. Science direct; 52(4):526.

- Burake W., Fesinmeyar M., Reed K., (2013): Family history as a predictor of asthma risk. American Journal of Preventive Medicine 24(2):160-9.

- Cogswell J., (2010): Influence of maternal atopy on atopy in the offspring. Clin Exp Allergy; 30(1):1-3.

- El-Deeb H., (2016 ): Effect of reflexology foot massage on fatigue level and leg cramps for patients on hemodialysis. Unpublished Doctorate Thesis, Faculty of Nursing, Alexandria University.

- El-Fadawy H., Abd Elhameed S., \& Hassnaen A., (2018): Effect of Acupressure on Physical and Psychological Health Status of Elderly with Knee Joint Osteoarthritis. IOSR Journal of Nursing and Health Science: 2320(7):42-54.

- El-Saadawy D., (2013): Effect of acupressure on dyspnea and fatigue among patients with chronic obstructive pulmonary disease. Journal of Education and Practice; 4(22): 1735-2222.

- Greenblatt R., Mansour O., Zhao E., \& Himes B., (2017): Gender specific determinants of asthma among U.S. adults. Asthma Research and Practice; 3(2): 254-64.

- Hasegawa K., Bittener J., Herrera V., Gabriel S., Camargo C., (2013): Adults with frequent hospitalization for acute asthma. ATS journal; 7(5):123-75.

- Jang A., Choi I., Nam H., Kweon S., Ho Son M., Park S., Kim D., Kim Y., \& Par C., (2014): The effect of Passive Smoking on Asthma Symptoms, Atopy, and Airway hyperresponsiveness. J Korean Med Sci; 19(2): 214-17.
- John H., (2013): Handbook of biological statistics. Available at http://udel.edu/mcdonald/statifishers.html. Retrieved on 2016.

- Kasper D., Fauci A., Longo D., Braunwald E., \& Hauser S., (2015): Harrison's Principles of Internal Medicine. $18^{\text {th }}$ ed. New York: McGrawHill: 1547-8.

- Keet C., Matsui E., McCormack M., \& Peng R., (2017): Urban residence, neighborhood poverty, race/ethnicity, and asthma morbidity among children on Medicaid. Journal of Allergy and Clinical Immunology; 140(3): 822-7.

- Kim S., Sim S., \& Cho H., (2017): Active, passive, and electronic cigarette smoking is associated with asthma in adolescents. Scientific report; 177(89):156-63.

- Kim S., Sim S., \& Cho H., (2018): Active and passive smoking impacts on asthma with quantitative and temporal relations: A Korean Community Health Survey. Scientific report; 86(14):52-75

- Koch R., (2017): 12-month prevalence of asthma among adults in Germany. Journal of Health Monitoring; 2(3):34-42.

- Lavietes M., (2015): The Interpretation of Dyspnea in the Patient with Asthma. Pulmonary medicine; 2015(5): 230-76.

- Launois C., Barbe C., Bertin E., Nardi J., \& Perotin J., (2012): The modified Medical Research Council scale for the assessment of dyspnea in daily living in obesity: a pilot study. BMC Pulmonary Medicine; 12(61); 1471-2466.

- Maa S., Hsu W., (2013): Acupressure improves the weaning indices of tidal volumes and rapid shallow breathing index in stable coma patients receiving mechanical ventilation: randomized controlled trial. Evid Based Complement Altern Med; 20 (13):1-10.

- Maa S., Tsou T., Wang K., \& Lee K., (2020): Self-administered acupressure reduces the symptoms that limit daily activities in bronchiectasis patients: Journal of clinical nursing; 16 (4): 794 -804.

- Mehtaa P., Dhapteb V., \& Kadam S., (2017): Contemporary acupressure therapy: Adroit cure for painless recovery of therapeutic ailments. Journal of Traditional and Complementary Medicine; 7(2): 251-63.

- Ngai S., \& Chan C., (2016): A short review of acupuncture and bronchial asthma: Western and Traditional Chinese Medicine Concepts. Hong Kong Physiotherapy Journal; 24(1): 28-38.

- Osborn K., Wraa C., \&Watson A., (2010): Medical Surgical Nursing.5th ed Boston: Pearson; 560.

- Pietiläinen K., Kaprio J., \& Borg P., (2010): Physical inactivity and obesity: A vicious circle. National library of medicine; 16(2): 409-14. 
- Pigantaro F., Bonini M., Forgoiene A., Melandri S., \& Usmani O., (2017): Asthma gender: The female lung. Pharmacological Research; 119(5): 384-39.

- Rambabu B., Chandran C., Prasad A., Manikyamba D., \& Kumari A., (2016): Study of prevalence and risk factors of bronchial asthma in urban and rural areas of Kakinada. J. Evolution Med. Dent. Sci; 5 (21): 2278-4748.

- Refaata S., \& Aref H., (2014): Acute asthma in emergency department, prevalence of respiratory and non-respiratory symptoms. Egyptian Journal of Chest Diseases and Tuberculosis; 63(4): 7716.

- Shaw D., Sousa A., \& Fowler S., (2015): Clinical and inflammatory characteristics of the European U-BIOPRED adult severe asthma cohort. Eur Respir J; 46: 1308-21.

- Statistical records of Alexandria Main University Hospital. (2018-2019): Alexandria, Egypt.

- Tageldin M., Wagih K., Maher O., (2015): Study the pattern of bronchial asthma among outpatient's clinic at Sohag and Akhmeem chest hospitals. Egyptian Journal of Chest Diseases and Tuberculosis; 64(2): 313-23.

- Tang W., Chen W., Yu C., Chang Y., Chen C., Wang C., Yang S., (2014): Effects of acupressure on fatigue of lung cancer patients undergoing chemotherapy: an experimental pilot study. Complement Ther Med; 22(4):581-91.

- Townsend E., Yim P., Gallos G., \& Emala C., (2012): Can We Find Better Bronchodilators to Relieve Asthma Symptom. Journal of Allergy; 20(12):321-45

- Trott p., \& Oei S., (2019): Acupuncture for Breathlessness in Advanced Diseases: A Systematic Review and Meta-analysis, JPSM; 59(2): $327-38$.

- Vagharseyyedin S., Salmabadi M., Bahrami H., \& Riyasi H., (2018): The impact of selfadministered acupressure on sleep quality and fatigue among patients with migraine: A randomized controlled trial. Complement Therapy Med; 203(18):388-92.

- Williams L., Helvoort H., Dekhuijzen R., Hees H., \& Heijdra Y., (2016): Respiratory constraints during activities in daily life and the impact on health status in patients with early stage COPD: a cross-sectional study. Primary Care Respiratory Medicine; 9(1):54-62.

- Zein J., Dweilk R.,Comhair S., Bleecker E., Moore W., Peters S., \& Gastro M., (2015): Asthma is more severe in older adult. PLoS ONE; 10(7):133-45. 